\title{
A New Technique for Transmission of Pre-Encoded MPEG VBR Video Using CBR Service*
}

\author{
John Lauderdale and Danny H. K. Tsang \\ eelauder@ee.ust.hk eetsang@ee.ust.hk \\ Department of Electrical and Electronic Engineering \\ The Hong Kong University of Science and Technology, Clear Water Bay, Kowloon, Hong Kong
}

\begin{abstract}
This paper presents the system issues involved with the transmission of pre-encoded VBR MPEG video using CBR service. Conventional wisdom suggests that lossless delivery of VBR video using CBR service requires bandwidth to be reserved at the peak rate resulting in low bandwidth utilization. We calculate the minimum rate at which bandwidth must be reserved on a network in order to provide continuous playback of an MPEG encoded video bitstream. Simulation results using the frame size traces from several pre-encoded MPEG bitstreams and several buffer sizes demonstrate that this "minimum reservation rate" is much lower than the peak rate when a modest playback buffer size is used, resulting in much higher bandwidth utilization. Procedures for performing connection setup and lossless realtime video playback between the video server and the client are outlined. Methods for incorporating VCR-like features such as "pause" and "fast forward/reverse" for Video-on-Demand (VoD) applications are presented.
\end{abstract}

Keywords Minimum Reservation Rate, VBR video, MPEG, continuous media, Video-on-Demand (VoD), video server, bandwidth allocation

\section{Introduction}

With the large scale deployment of Broadband ISDN, digital video is foreseen to become an increasingly important type of network traffic. Much of this digital video traffic is expected to comply to the MPEG video compression standard[5]. Most MPEG compliant video coder-decoders (codecs) produce either Constant Bit Rate (CBR) or Variable Bit Rate (VBR) streams. CBR codecs keep the output bit rate constant by changing the amount of discarded video information in each frame ${ }^{1}$, thus producing video with frame-to-frame quality variations. Allocating network resources for CBR video transmission is very easy since the bit rate does not change. VBR video codecs produce constant-quality video by keeping

*Supported by Hongkong Telecom Institute of Information Technology grant HKTITT93/94.EG01

${ }^{1}$ In order to reduce the video bitstream rate, either the number of quantization levels of the DCT coefficients is reduced, or the high frequency DCT coefficients are not coded. See $[1,4,5]$ for details about MPEG rate control. the amount of discarded video information in each frame constant; however, VBR streams may require the output bit rate to change from frame to frame which increases network resource allocation complexity. Frame-to-frame rate variations with VBR MPEG video may be very high, up to an order of magnitude, due to video content as well as the type of frame in an MPEG sequence. ${ }^{2}$

Adaptive rate controlling techniques change the rate of the codec (and the quality of the resulting video) based on the network resources available[9, 10]. Most of these adaptive techniques require realtime encoding which may be very computation intensive if motion compensated prediction is used to reduce temporal redundancies in MPEG's P-frames and B-frames.

In order to avoid loss of pre-encoded VBR MPEG bitstream data, the network provider can simply allocate bandwidth equivalent to the peak rate, which is the maximum frame size divided by the frame period. While peak-rate bandwidth allocation is very simple, it wastes bandwidth. Alternatively, bandwidth can be dynamically allocated during each frame interval, which results in high bandwidth utilization, but the frequent rate changes may make network resource allocation more difficult.

"Piecewise-constant bandwidth scheduling" [3] can be used for delivery of pre-encoded video with known playback buffer size. It breaks a long MPEG sequence into several short segments and calculates a constant bandwidth required during each segment such that the playback buffer does not overflow or underflow. Network resource allocation is simplified because the number of rate changes is reduced. This method can achieve high bandwidth utilization; however, it does not work well for Video-on-Demand applications which give the user Temproal Access Control (TAC) with VCR-like features such as "pause", "fast forward", and "fast reverse" because these features shift the time base of the movie. Since bandwidth is reserved for each segment, and each segment is associated with a particular time interval, bandwidth needs to be re-scheduled and re-reserved when there is a shift in the time base.

Asynchronous Transfer Mode (ATM) networks are de-

\footnotetext{
${ }^{2}$ MPEG defines three frame types: I, P, and B. Predicted P-frames are usually smaller than Intra coded I-frames and larger than Bidirectionally interpolated B-frames.
} 
signed to accommodate many types of network traffic such as data, voice, and video. ATM Adaptation Layers (AALs) have been defined to accommodate service classes with different attributes such as the timing relationship required between the source and destination, whether the bit rate is constant or variable, and whether the connection mode is connectionoriented or connectionless[8]. AAL1 and AAL2 have been designed for connection oriented, delay-sensitive, CBR traffic and VBR traffic, respectively. This paper considers the delivery of pre-encoded VBR MPEG bitstreams using AAL1 (CBR service), which provides circuit emulation. AAL1 is easier for the network to schedule and is better defined at this time than AAL2. Quality of Service (QoS) guarantees for CBR service specify the transmission rate, the average transmission delay, the maximum jitter and the cell loss probability.

The remainder of this paper is organized as follows: Section 2 introduces the minimum reservation rate and presents a system model for delivery of an MPEG bitstream from a video server to an MPEG decoder through a network and video playback buffer. Section 3 uses frame size traces from several MPEG bitstreams to demonstrate the tradeoff between the minimum reservation rate and the playback buffer size. Section 4 details resource management procedures for connection establishment and interactive video playback. Section 5 concludes.

\section{System Model}

The system model consists of a video server, an ATM network, a playback buffer, and an MPEG decoder as shown in Figure 1. The $i^{\text {th }}$ frame of a pre-encoded MPEG bitstream has $a_{i}$ bits $(i=1,2, \ldots, n)$, where $n$ is the number of frames in the movie. The bitstream is decoded at a rate of $1 / \tau$ frames per second, where $\tau$ seconds is the frame period.

$T_{i}$, the time interval for each frame $i$, is constant since the frame rate of video remains constant, (i.e. $T_{i}=\tau$ for all $i$ ). The decoder removes $a_{i}$ bits of the MPEG bitstream from the playback buffer during the $i^{t h}$ frame interval, [ $\left.\left.i-1\right) \tau, i \tau\right]$.

The minimum reservation rate, $R^{*} \mathrm{bits} / \mathrm{sec}$, is the minimum rate at which bandwidth must be reserved in the ATM network to ensure that the playback buffer does not starve (or underflow). It is precomputed for a small number of buffer sizes $\tilde{B}_{P}$ based on the frame sizes of the pre-encoded bitstream and stored in a lookup table. The proof for computing $R^{*}$ is detailed and a computationally efficient algorithm is presented in [6], which shows that

$$
R^{*}=\max _{1 \leq i \leq j \leq n} \frac{a_{i}+\cdots+a_{j}-B_{P}}{T_{i}+\cdots+T_{j}},
$$

where $a_{i}+\cdots+a_{j}$ is the sum of the frame size of the $i^{t h}$ to $j^{t h}$ frames, $T_{i}+\cdots+T_{j}$ is the frame period of the $i^{\text {th }}$ to $j^{\text {th }}$ frames, and $B_{P}$ is the size of the playback buffer. In [2] we show how the structure of MPEG video can be exploited to simplify the direct computation of $R^{*}$. We also show how bandwidth can be saved when multiple bitstreams are considered together when computing $R^{*}$.
The video server requests $R^{*}$ bits/sec from the network. The network's Call Admission Control (CAC) algorithm accepts or rejects the request based on the available resources. If the connection is accepted, the ATM network will provide CBR service with guaranteed transmission rate $R^{*}$ bits/second, average delay $D$ seconds and maximum delay jitter $\Delta$ seconds. Here we assume lossless transmission since the cell loss probability due to buffer overflow is zero for CBR service and the probability of cell loss due to transmission error is negligibly small. To simplify network resource management, it is assumed that bandwidth can not be renegotiated once a connection is set up.

The rate at which data is received by the playback buffer during the $i^{t h}$ frame interval is the buffer input rate, $I_{i}$. This is also the rate at which the server sends out data during the time interval $[(i-1) \tau-D, i \tau-D]$. It is calculated using the algorithm presented in [2] and is the highest feasible rate which does not cause the playback buffer to overflow and does not exceed the negotiated bandwidth (i.e. $I_{i} \leq R^{*}$ for all $i$ ). Therefore, although $R^{*}$ bits/second are reserved on the network, the actual rate at which data is transmitted is sometimes less than $R^{*}$, causing some of the reserved bandwidth to be unutilized ${ }^{3}$. The reserved but unused bandwidth could be made available to transmit Available Bit Rate (ABR) traffic, if any.

Let $b(t)$ be the buffer occupancy at time $t$. The buffer occupancy varies continuously between 0 and the playback buffer size $\left(0 \leq b(t) \leq B_{P}\right)$. The "minimum buffer pre-load" for the minimum reservation rate is defined in [6] as follows. Given a feasible $R^{*}$ with $b(0)=B_{P}$, find the smallest value $b^{*}$ such that $R^{*}$ is still feasible with $b(0)=b^{*}$. In order to reduce the initial playback delay, the "minimum buffer pre-load" is calculated using the algorithm presented in [2].

\section{The Minimum Reservation Rate for an MPEG bitstream}

The frame size traces from several 10,000 frame MPEG bitstreams[7] were used to evaluate the tradeoff between the size of the playback buffer, $B_{P}$, and the minimum reservation rate, $R^{*}$. The amount of computation required for solving the problem was reduced by using the "I-frame only" method which is detailed in [2]. The buffer sizes are evaluated at $B_{P}=10^{4} * 2^{k}$ bits where $k=0,1,2, \ldots, 13$. The MPEG-1 encoder parameters are shown in Table 1 . The statistics for each bitstream are shown in Table 2, which also shows $R^{*}$ and the bandwidth utilization for selected buffer sizes. The bandwidth utilization is calculated by dividing the mean rate of each bitstream by the reservation rate for each buffer size. Dividing the mean frame size by the maximum frame size for each bitstream shown in Table 2 shows that allocating bandwidth at the peak rate results in bandwidth utilization of less than $20 \%$. For the bitstreams used in our simulations, whose parameters are shown in Table 1 , when a modest buffer size

\footnotetext{
${ }^{3}$ Bandwidth utilization is the ratio of used bandwidth and reserved bandwidth.
} 


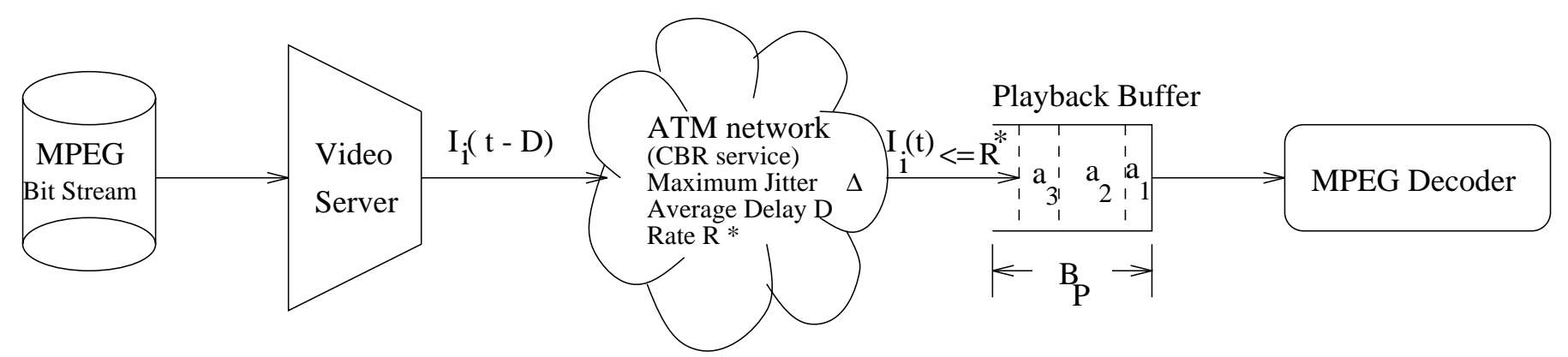

Figure 1: System model for video-server application.

\begin{tabular}{|c|c|c|c|c|c|c|c|c|c|c|}
\hline \multirow[b]{3}{*}{ Bit stream } & \multirow{2}{*}{\multicolumn{4}{|c|}{$\begin{array}{l}\text { Bit stream statistics } \\
\left(* 10^{3} \mathrm{bits} / \mathrm{sec}\right)\end{array}$}} & \multirow{2}{*}{\multicolumn{3}{|c|}{$\frac{R^{*}\left(* 10^{3} \text { bits } / \mathrm{sec}\right)}{B_{P}\left(* 10^{4} \text { bits }\right)}$}} & \multirow{2}{*}{\multicolumn{3}{|c|}{$\frac{\overline{\text { bandwidth utilization }}}{B_{P}\left(* 10^{4} \text { bits }\right)}$}} \\
\hline & & & & & & & & & & \\
\hline & $\operatorname{Max}$ & Mean & Min & Std. Dev. & $2^{0}$ & $2^{4}$ & $2^{10}$ & $2^{0}$ & $2^{4}$ & $2^{10}$ \\
\hline MTV & 6876 & 819 & 24.5 & 784 & 5630 & 3056 & 1236 & 0.1455 & 0.2681 & 0.6626 \\
\hline News & 5191 & 458 & 37.2 & 586 & 4891 & 1305 & 471 & 0.0937 & 0.3511 & 0.9724 \\
\hline James Bond & 4791 & 753 & 62.6 & 806 & 4491 & 1617 & 1013 & 0.1678 & 0.4661 & 0.7440 \\
\hline Jurrassic Park & 2507 & 382 & 26.4 & 390 & 2207 & 1202 & 382 & 0.1728 & 0.3173 & 1 \\
\hline Star Wars & 2239 & 298 & 8.4 & 376 & 1940 & 767 & 312 & 0.1533 & 0.3876 & 0.9505 \\
\hline
\end{tabular}

Table 2: Statistics for MPEG bitstreams, $R^{*}$, and bandwidth utilization.

\begin{tabular}{|ll|}
\hline Picture Rate & 30 frames per second \\
GOP size & 12 \\
encoder input & 384 x 288 pel \\
motion vector search & 'Logarithmic' / 'Simple' \\
color format & YUV $(4: 1: 1$, resolution of 8 bits $)$ \\
reference frame & 'Original' \\
quantization values & I=10, P=14, B=18 \\
slices & 1 \\
pattern & IBBPBBPBBPBB \\
vector/range & half pel / 10 \\
\hline
\end{tabular}

Table 1: MPEG bitstream encoder parameters

of $10^{4} * 2^{10}$ bits (about 1.3 Megabytes) is used, the bandwidth utilization for the "News", "Jurrassic Park", and "Star Wars" bitstreams are above 95\%, while "MTV" and "James Bond" have bandwidth utilization above $66 \%$. The minimum reservation rate and bandwidth utilization for all playback buffer sizes are shown in Figure 2, which uses a semi-log scale. Figure 2 shows the minimum reservation rate is much lower than the peak rate when a modest playback buffer size is used, resulting in much higher bandwidth utilization.

\section{Procedures for Resource Manage- ment}

This section presents procedures that can be used to design communication protocols between the video server, the network, and the client. The "connection establishment" procedure negotiates acceptable Quality of Service (QoS) for the movie selected. The "video playback" procedure guarantees continuous video playback as long as the QoS is guaranteed. The video server calculates the output rates by simulating an undelayed version of the playback buffer occupancy. The ac- tual playback buffer occupancy is a delayed version of the simulated buffer occupancy since the simulated playback buffer occupancy does not account for network delay, which is guaranteed to be constant (within the bounds of the delay jitter).

The simulated and actual playback buffers become out of synchronization when interactive TAC features such as "pause", "fast forward", and "fast reverse" are used. The "pause" procedure details how the simulated and actual buffers can be synchronized in order to avoid playback buffer overflow or underflow when playback is resumed. The "fast forward/reverse" procedure shows how the structure of MPEG can be used to skip frames, how the reserved bandwidth can be fully exploited, and how to resynchronize the simulated and actual playback buffers when playback is resumed. The network delay, $D$, affects only the initial playback delay and the playback delay due interactive TAC features.

\subsection{Video Connection Establishment}

1. The client requests a movie and informs the video server of the actual playback buffer size, $B_{P}$.

2. If the video server is overloaded and can not accommodate the request, the request is immediately rejected.

3. The video server looks up the pre-computed minimum reservation rate, $R^{*}$, from the table based on $\tilde{B}_{P}$, the largest playback buffer size that does not exceed $B_{P}$.

4. The maximum allowable jitter is calculated:

$$
\Delta_{\max }=\frac{B_{P}-\tilde{B}_{P}}{2 R^{*}}
$$

5. The video server requests $R^{*}$ bits/second with maximum jitter $\Delta_{\max }$ from the network. 

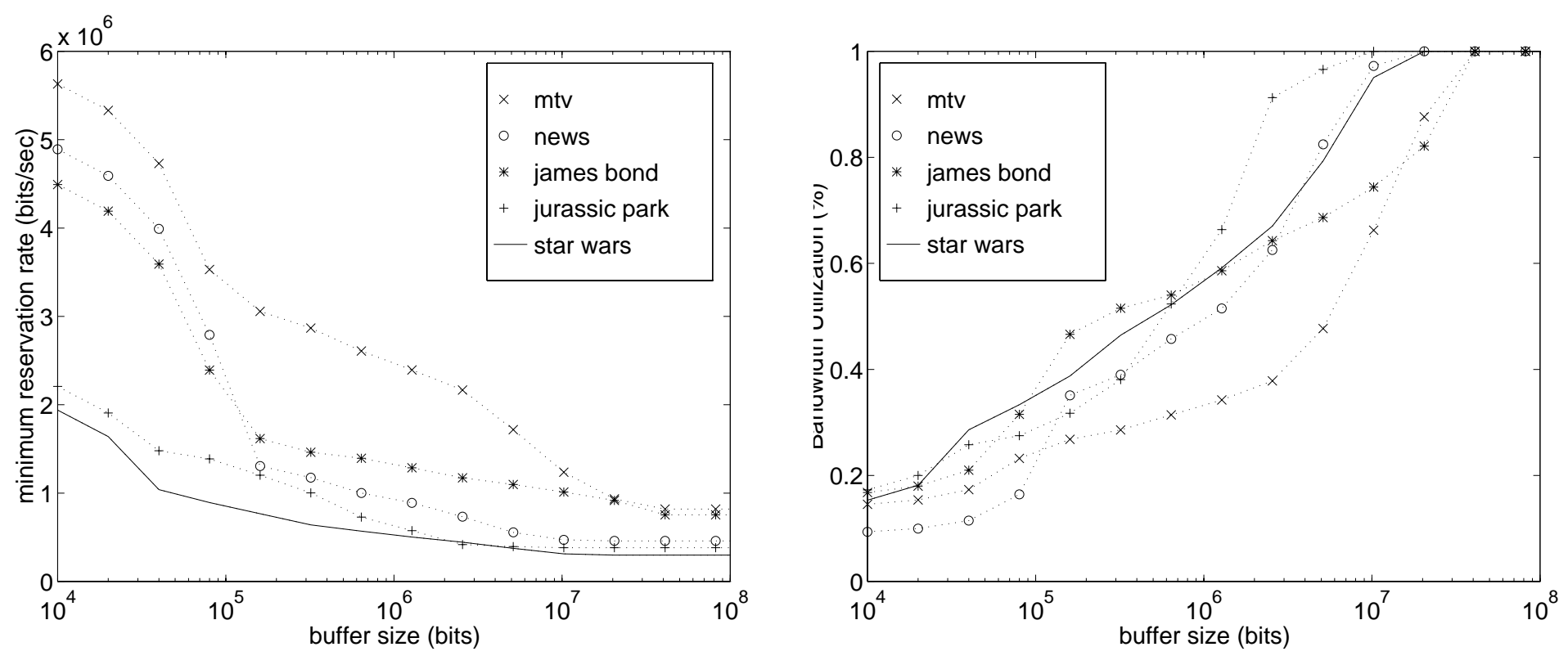

Figure 2: $R^{*}$ and bandwidth utilization vs. $P_{B}$ for 10,000 frame MPEG bitstreams.

6. If the network can satisfy the requirements, it accepts the request, reserves bandwidth, and specifies the QoS parameters including delay, jitter, and cell loss probability.

7. If the network cannot satisfy the jitter requirement, it informs the server. The server then uses the next-lowest buffer size value in the table to lookup a new $R^{*}$. It recalculates the jitter requirement which will most likely be less stringent because the numerator in Equation 2 will most likely increase faster than the denominator when $\tilde{B}_{P}$ is decreased and $R^{*}$ is increased. The new $R^{*}$ and $\Delta_{\max }$ are negotiated with the network.

8. If the network cannot satisfy the bandwidth requirement, the network rejects the video server's request and the video server rejects the client's request.

\subsection{Video Playback}

1. The video server calculates the minimum buffer preload, $b_{0}$, and informs the client. In order to minimize the playback delay, $b_{0}$ data is sent at the maximum rate $R^{*}$

2. When the client receives $b_{0}$ data, it begins playing back the movie which removes $a_{i}$ bits from the playback buffer during the $i^{\text {th }}$ frame interval.

3. The video server simulates the playback buffer occupancy and sends data at rate $I_{i}$, which is the largest transmission rate possible for the $i^{t h}$ time interval such that the simulated playback buffer does not overflow.

\subsection{Video "pause" and "resume play"}

1. When the user presses "pause", the client stops decoding successive frames and notifies the video server of the frame being displayed. Since video data are still in transit, the client buffer continues to fill (and may overflow since video data are not being removed from the buffer).

2. Once the video server receives the "pause" message, it finishes sending the current frame but does not begin sending the next frame until a "resume play" signal is received.

3. When the user presses "resume play", the client notifies the video server of the last completely received frame and the buffer occupancy inclusive of all completely received frames.

4. The client removes all incompletely received frames from the buffer.

5. Upon receiving the "resume play" signal, the server updates the buffer occupancy and resumes playback. The first frame sent is a tagged version of the next frame which the client has not completely received. Tagging the first frame is necessary in order to distinguish it from the duplicate frame which may be in transit at the time "resume play" is pressed.

6. The client resumes video playback immediately upon receiving the beginning of the tagged frame. The calculated and actual buffers are resynchronized.

\subsection{Video "Fast Forward/Reverse"}

Since MPEG video coding uses both interframe and intraframe coding techniques, fast forward and fast reverse can be performed by displaying only intra-coded I-frames successively, thereby skipping B-frames and P-frames. The "speed" of the fast forward and reverse is determined by the number of I frames that are skipped. The video playback procedure 
guarantees continuous video playback when $R^{*}$ bits/second are reserved; however, continuous playback is not guaranteed for "fast forward/reverse", which change the frame playback sequence. Since I-frames are typically much larger than Pframes or B-frames, and since the negotiated maximum rate at which data can be sent is $R^{*}$ bits/sec, it may be necessary to substantially reduce the rate at which I-frames are displayed.

The "fast forward" procedure, detailed below, attempts to minimize the playback delay when "resume play" is pressed by not re-sending I-frames which are already in the playback buffer. This method may require complicated playback buffer memory management since frames are sent out of order. A simpler method, not detailed, is to empty the playback buffer when "resume play" is pressed, then resume playback by sending all frames in order. This simpler method, which increases playback delay, may be used for "fast forward" but must be used for "fast reverse" since the playback direction changes.

1. When the user presses "fast forward", the client skips to and successively displays all completely received Iframes, discarding all P-frames and B-frames. The video server is notified of the last displayed I-frame number and the number of I-frames to skip.

2. Once the video server receives the "fast forward" message, it sends I-frames at the maximum allowable rate, $R^{*}$ bits/second. Although when only I-frames are sent, buffer underflow is much more likely than buffer overflow, buffer overflow may be prevented by adjusting the transmission rate as is done during video playback.

3. The client displays the I-frames as they are received. The rate at which frames are displayed will most likely drop because the rate required to continuously display successive I-frames at the normal frame rate would most likely require more than $R^{*}$ bits/second. Although the frame rate drops, the apparent playback speed can be increased by increasing the number of skipped I-frames.

4. When "resume play" is pressed, the client sends the video server the frame number of the currently displayed I-frame and the frame number of the last completely received I-frame currently in the playback buffer.

5. When the video server receives the "resume play" signal, it sends the client a new minimum buffer preload value, which is adjusted to reflect the I-frames already in the playback buffer. The number of I-frames to keep are also sent.

6. Upon receiving the minimum buffer preload value and the number of I frames to keep, the client clears any unnecessary I-frames from the buffer.

7. Since the I-frames are already in the playback buffer, only the remaining $\mathrm{P}$-frames and $\mathrm{B}$-frames are sent at rate $R^{*}$ until data equivalent to the minimum buffer preload has been sent. The video server then resumes sending video data at rate $I_{i}$.
8. Movie playback resumes when the client playback buffer receives video data equivalent to the minimum buffer preload.

\section{Conclusion}

This paper demonstrated how pre-encoded VBR MPEG video can be efficiently and losslessly transmitted using CBR service for Video-on-Demand applications. When the MPEG bitstream frame sizes and the playback buffer sizes are known, the minimum reservation rate can be calculated. When a modest playback buffer size is used, the minimum reservation rate has been shown to be much lower than the peak rate, resulting in much higher bandwidth utilization. Resource management procedures for connection setup, video playback, and VCR-like features have been outlined to assist in implementation and protocol design. Our future research in this area may involve implementation of this method on our ATM network testbed.

\section{References}

[1] Simon S. Lam, Simon Chow, and David K.Y. Yau, "An Algorithm for Lossless Smoothing of MPEG Video," Proceedings of $A C M$ SIGCOMM'94, Vol. 24 No. 4 October 1994, pp. 281-293.

[2] John Lauderdale "Variable Bit Rate Video Transmission over ATM Networks" M.Phil Thesis, Chapter 3 "The Minimum Reservation Rate Problem for the Transmission of MPEG VBR Video," The Hong Kong University of Science and Technology, Dept. of Electrical and Electronic Engineering. August 1995. http://www.ee.ust.hk/ ${ }^{\sim}$ ustatm/index.html

[3] John Lauderdale, Danny H.K. Tsang. "Bandwidth Scheduling of Prerecorded VBR video for ATM networks," presented at IEEE ATM Workshop, Washington D.C., October 1995.

[4] D. LeGall. "MPEG: A video compression standard for multimedia applications," CACM, 34(4):46-58, April 1991.

[5] Coding of moving pictures and associated audio, November 1991. SC29/WG11 committee (MPEG) draft submitted to ISOIEC/JTC1 SC 29

[6] Nimrod Megiddo, Moni Naor, David P. Anderson. "The Minimum Reservation Rate Problem in Digital Audio/Video Systems," Proceedings of the 2nd Isreal Symposium on Theory and Computing Systems, June 1993, pp. 43-8.

[7] Oliver Rose. "Statistical properties of MPEG video traffic and their impact on traffic modeling in ATM systems," University of Wuerzburg. Institute of Computer Science Research Report Series. Report No. 101. February 1995. The VBR MPEG video traces used in this research were obtained via anonymous ftp from ftpinfo3.informatik.uni-wuerzburg.de in the /pub/MPEG directory.

[8] David E. McDysan, Darren L. Spohn, ATM : theory and application, McGraw-Hill, 1995

[9] P. Pancha and M. El Zarki. "Bandwidth requirements of variable bit rate MPEG sources in ATM networks," Proceedings of INFOCOM'93 pages 902-909, March 1993.

[10] H. Kanakia, P. Mishra, and A. Reibman. "An adaptive congestion control scheme for real-time packet video transport," Proceedings of ACM SIGCOMM'93 pages 20-31, Sept 1993. 